\title{
The role of Bcl-2 family member BNIP3 in cell death and disease: NIPping at the heels of cell death
}

\author{
TR Burton ${ }^{1,2}$ and SB Gibson ${ }^{\star, 1,2}$
}

$\mathrm{Bcl}-2$ nineteen-kilodalton interacting protein (BNIP3) is a BH-3-only Bcl-2 family member whose expression levels increase during stress such as hypoxia through hypoxia-inducing factor-1-dependent or -independent mechanisms. When BNIP3 expression is induced, it localizes to the mitochondria and triggers a loss of membrane potential, and an increase in the reactive oxygen species production, which often leads to cell death. Cells under normal growth conditions suppress BNIP3 expression through transcriptional repression. There is considerable debate in the literature regarding what type of cell death is induced by BNIP3. It has been observed that BNIP3 could induce necrosis, autophagy and/or apoptosis. In contrast, other studies indicate that BNIP3 could promote cell survival. Besides its cell death regulation, BNIP3 plays a key role in the pathogenicity of many diseases. In cardiac infarction, loss of BNIP3 expression has been shown to reduce the number of damaged cardiomyocytes after ischemia and reperfusion. BNIP3 expression also plays an important role in the deregulation of cell death in many cancers. In this review, we will discuss the different and often contradictory mechanisms of BNIP3 regulation of cell death and the role that BNIP3 may play in diseases.

Cell Death and Differentiation (2009) 16, 515-523; doi:10.1038/cdd.2008.185; published online 9 January 2009

$\mathrm{Bcl}-2$ nineteen-kilodalton interacting protein (BNIP3) belongs to the $\mathrm{Bcl}-2$ homology domain (BH3)-only $\mathrm{Bcl}-2$ family members because it only contains a putative $\mathrm{BH} 3$ domain. ${ }^{1}$ The other major domains found in BNIP3 are the PEST domain that targets BNIP3 for degradation, a conserved domain that is conserved from Caenorhabditis elegans to humans and a transmembrane (TM) domain, which targets BNIP3 to the mitochondria (Figure 1). ${ }^{2} \mathrm{BH} 3$-only containing proteins act as rheostats regulating apoptosis through their $\mathrm{BH} 3$ domain by binding to antiapoptotic Bcl-2 family members. However, BNIP3 differs from these members, as its $\mathrm{BH} 3$ domain fails to interact with antiapoptotic $\mathrm{Bcl}-2$ family members. ${ }^{3}$ Furthermore, deletions of the $\mathrm{BH} 3$ domain fail to affect the ability of BNIP3 to induce cell death. ${ }^{4}$ Unlike other BH3-only family members, BNIP3 interacts with Bcl-2 and $\mathrm{Bcl}-\mathrm{X}_{\mathrm{L}}$ through its $\mathrm{TM}$ domain and $\mathrm{N}$ terminus (amino acids 1-49). ${ }^{3}$ Deletion of the TM domain blocks the ability of BNIP3 to induce cell death. ${ }^{4}$ BNIP3 migrates at 30 and $60 \mathrm{kDa}$, indicating that BNIP3 is a protein that forms homodimers. This homodimerization is primarily through the TM domain of BNIP3. The unique structure of the TM domain suggests that BNIP3 dimers could act as proton channels in the outer mitochondrial membrane increasing ion conductance. ${ }^{5}$ Serine
172 and histidine (His) 173 are residues that are present in the dimerization interface of BNIP3. These residues interact by hydrogen bonds and are essential for dimer formation. ${ }^{6}$ Furthermore, mutation of the His 173 to alanine completely abrogated the ability of BNIP3 to induce cell death. ${ }^{7} \mathrm{Bcl}-2$ and $\mathrm{Bcl}-\mathrm{X}_{\mathrm{L}}$ can compete for binding to the TM domain, which blocks BNIP3 homodimerization and abrogates the ability of BNIP3 to induce cell death (Figure 2). ${ }^{3}$ Thus, BNIP3 is a $\mathrm{BH} 3-$ only Bcl-2 family member with unique properties.

Another protein called NIX or BNIP3L was identified using sequence homology to be related to BNIP3 (56\% identity). ${ }^{8}$ NIX includes a PEST, BH3 and TM domains that are similar to BNIP3. $^{2}$ In addition, hypoxia induces NIX and BNIP3 expression. NIX also localizes to mitochondria and induces cell death similar to BNIP3 ${ }^{2}$ Furthermore, NIX is upregulated in heart muscle following ischemic injury and in human breast tumors similar to BNIP3. ${ }^{9}$ Thus, NIX is a BH-3-only Bcl-2 family member with similar functions to BNIP3.

\section{How is BNIP3 expression regulated?}

The expression of BNIP3 needs to be tightly regulated because overexpression induces cell death. Stabilization of

\footnotetext{
${ }^{1}$ Manitoba Institute of Cell Biology, Winnipeg, Manitoba, Canada and ${ }^{2}$ Department of Biochemistry and Medical Genetics, University of Manitoba, Winnipeg, Manitoba, Canada

${ }^{*}$ Corresponding author: SB Gibson, Manitoba Institute of Cell Biology, 675 McDermot Avenue, Winnipeg, Manitoba, Canada R3E 0V9. Tel: + 204 787 2051;

Fax: + 204787 2190; E-mail: gibsonsb@cc.umanitoba.ca

Keywords: BNIP3; apoptosis; ischemia; hypoxia; autophagy; cancer

Abbreviations: $\Delta \psi \mathrm{m}$, mitochondrial membrane potential; 5-FU, fluorouracil; ACAA2, acetyl-coenzyme A acyltransferase 2; AR, androgen receptor; Aza-dC, 5-aza-2deoxycytidine; $\mathrm{BH} 3$, bcl-2 homology domain; BNIP3, bcl-2 nineteen-kilodalton interacting protein; DCIS, ductal carcinoma in situ; EGF, epidermal growth factor; EM, electron microscopy; GBM, glioblastoma multiforme; HCC, hepatocellular carcinoma; HDAC, histone deacetylase; HIF-1, hypoxia-inducing factor-1; His, histidine; I/R, ischemia-reperfusion; KO, knock-out; LC3, light chain 3; MM, multiple myeloma; mTOR, mammalian target of rapamycin; NF $\kappa$ B, nuclear factor kappa B; PDAC, pancreatic ductal adenocarcinoma; PT, permeability transition; Rb, retinoblastoma; ROS, reactive oxygen species; TM, transmembrane domain

Received 09.9.08; revised 28.10.08; accepted 28.10.08; Edited by C Borner; published online 09.1.09
} 
Multidomain

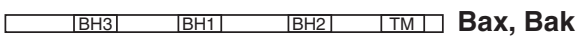

BH3-only

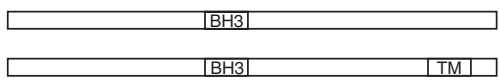

\begin{tabular}{|l|l|l|l|l|l|l|l|l|}
\hline PEST & CD
\end{tabular}

Figure 1 Schematic diagram of the pro-cell death Bcl-2 family members. The multidomain proteins share Bcl-2 homology 1, 2 and 3 domains (BH1-3). The BH3only members contain a $\mathrm{BH} 3$ region of homology. Most members contain a transmembrane (TM) domain that functions in membrane localization. BNIP3 is a $\mathrm{BH} 3-$ only member of the $\mathrm{Bcl}-2$ family that contains a $\mathrm{BH} 3$ domain, TM domain, PEST domain that controls protein degradation and a conserved domain (CD) that is conserved from C. elegens to humans

the hypoxia-inducing factor-1 (HIF-1)- $\alpha$ under hypoxic conditions allows this transcription factor to induce expression of multiple genes. One of the most abundant genes induced by HIF-1 is bnip3. BNIP3 mRNA and protein levels are upregulated due to a HIF-1-responsive element that occurs in the proximal promoter for bnip3 that binds to HIF-1, inducing BNIP3 expression. ${ }^{10}$ This element is highly conserved between the rat, mouse and human genomic sequence. Signaling through the glucocorticoid receptor also increases BNIP3 expression in neurons, suggesting that besides HIF-1 other transcription factors could regulate BNIP3 expression under hypoxia conditions. ${ }^{11}$ Under hypoxia, stabilization of p53 also occurs, but BNIP3 upregulation under hypoxia was found to be independent of p53. ${ }^{12-14}$ Nitric oxide also induces BNIP3 expression through activation of RAS signaling pathway in macrophages and enterocytes. ${ }^{15}$ Treating cells with toxins that induce cell death, such as arsenic trioxide and cyanide, or with cellular products ceramide and amyloid- $\beta$ also induce BNIP3 expression mediated by HIF-1 activation. ${ }^{16,12-14}$ In addition, transcription factor E2F-1 binds to the bnip3 promoter and induces its expression in rat ventricular myocytes. ${ }^{17}$ Therefore, it is becoming apparent that many cellular stresses such as hypoxia or toxins lead to increased BNIP3 expression.

To survive in normal growth conditions, cells require mechanisms to repress BNIP3 expression. The tumor suppressor retinoblastoma $(\mathrm{Rb})$ has been shown to attenuate the expression of BNIP3 under hypoxia and reduce the amount of hypoxia-induced cell death. ${ }^{18}$ This is mediated through the binding of transcription factor E2F to the bnip3 promoter in mouse fibroblasts, hepatocytes and tumor cells. ${ }^{18}$ BNIP3 expression is repressed in rat ventricular myocytes through the transcription factor nuclear factor kappa $\mathrm{B}(\mathrm{NF} \kappa \mathrm{B})$ binding to the bnip3 promoter. ${ }^{19}$ This is due to recruitment of histone deacetylase (HDAC)-1 by $\mathrm{NF}_{\kappa} \mathrm{B}$ to the promoter leading to suppression of hypoxia-induced cell death. ${ }^{20}$ Concurrently, in p65-l- cells (NF $\kappa$ B subunit), HDAC1 fails to repress bnip3 gene transcription, revealing the importance of $\mathrm{NF}_{\kappa} \mathrm{B}$ in the regulation of BNIP3 in myocytes. ${ }^{20}$ Thymidine phosphorylase and S100A4 also inhibit BNIP3 expression. $^{21,22}$ This suggests that there are multiple proteins

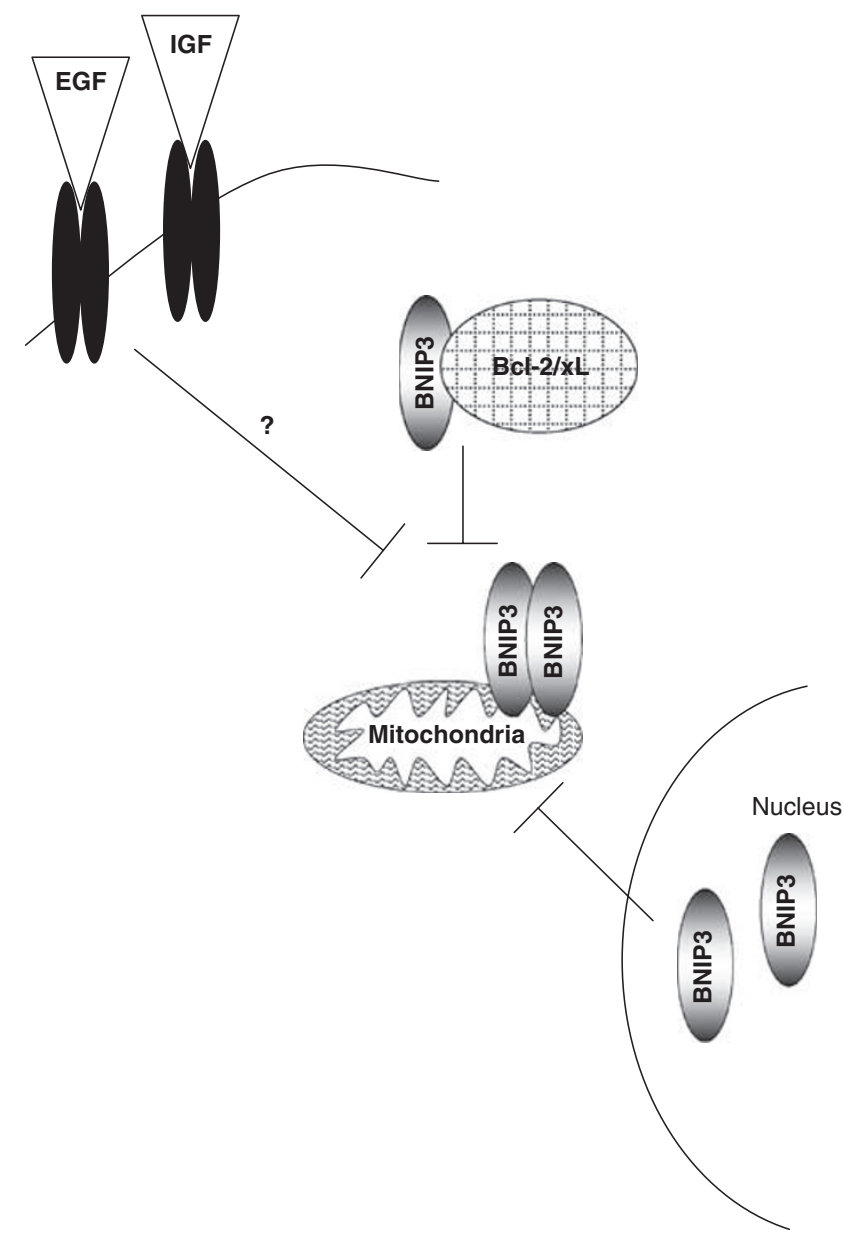

Figure 2 Mechanisms that block BNIP3-induced cell death. Bcl-2 and Bcl-xL bind to BNIP3 and block its ability to dimerize and induce cell death through the mitochondria. EGF and IGF signaling blocks BNIP3-induced cell death. Finally, nuclear-localized BNIP3 is unable to interact with the mitochondria and induce cell death

repressing BNIP3 expression, preventing BNIP3-induced cell death under normal conditions.

\section{Post-translational modification of BNIP3}

Regulation of BNIP3 by the ubiquitin-proteasome pathway is not clear. BNIP3 has a known PEST domain, but a link between its degradation and function is unknown. One study has identified that BNIP3 is phosphorylated and that elevation of phospho-BNIP3 correlated with BNIP3-induced cell death. ${ }^{23}$ BNIP3 could also be modified by $O$-linked $\mathrm{N}$-acetylglucosamine in lung tumor cells, which attenuates the ability of BNIP3 to induce cell death. ${ }^{24}$ Further work is required to discover what mechanisms control post-translational modification of the BNIP3 protein, where the modifications occur and what role they play in altering the function of BNIP3.

\section{Does BNIP3 induce different types of cell death?}

There are three main types of cell death: necrosis, autophagy and apoptosis. Necrosis is characterized by mitochondrial 


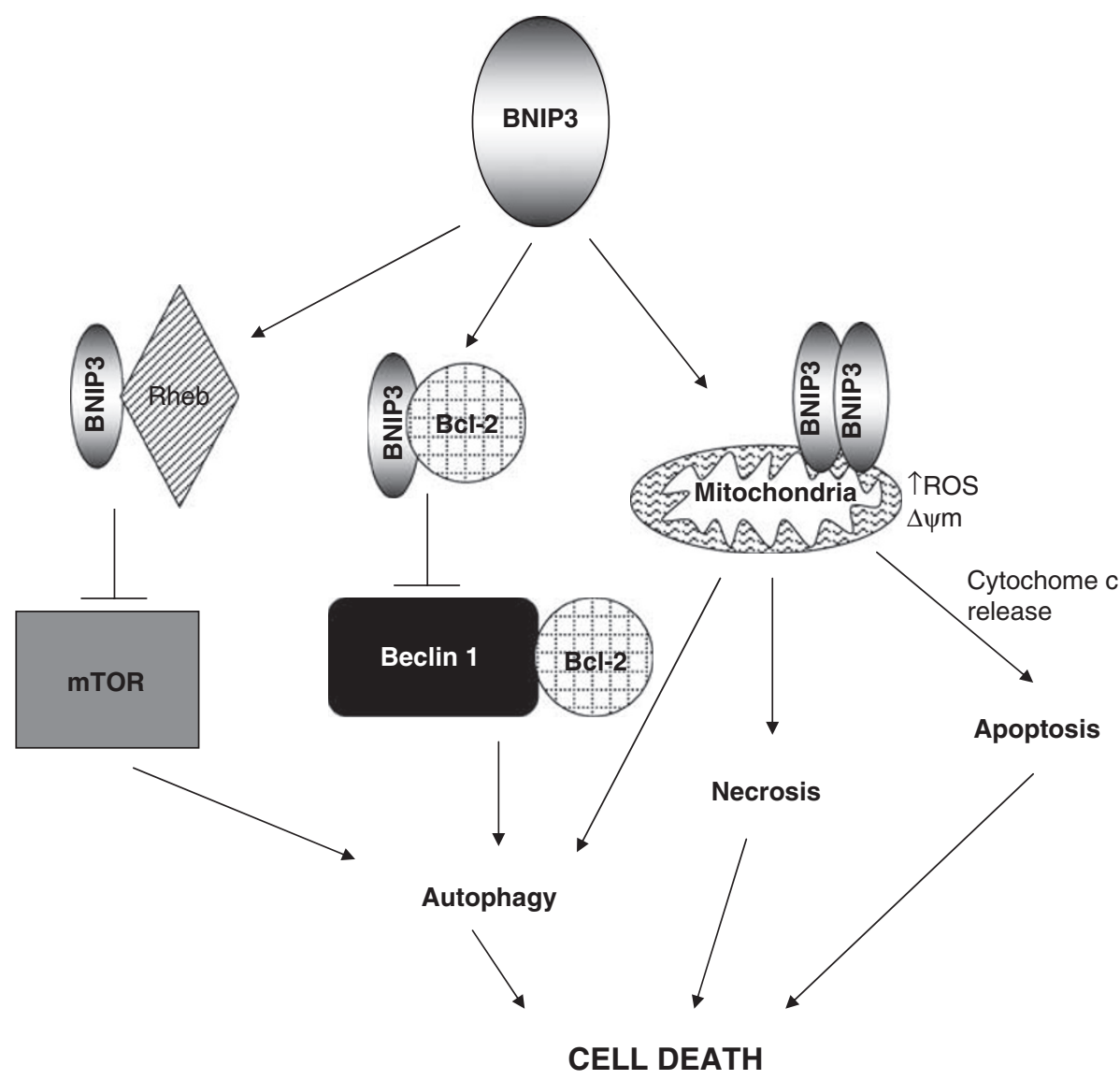

Figure 3 Mechanisms of BNIP3-induced cell death. BNIP3 dimerizes, inserts in the mitochondrial membrane and can induce cell death through apoptotic, necrotic and autophagic pathways. BNIP3 can induce autophagic cell death by competing with Beclin 1 for binding to Bcl-2. When Beclin 1 is not bound to Bcl-2, it is able to induce autophagic cell death. BNIP3 can also bind to Rheb and block its ability to activate mTOR, leading to autophagic cell death

swelling, permeability transition (PT) pore opening, loss of mitochondrial membrane potential $(\Delta \psi \mathrm{m})$ and reactive oxygen species (ROS) production. ${ }^{25}$ This leads to cellular rupture and release of intracellular contents into the extracellular space. Autophagy is an intracellular degradation system normally used for protein recycling and organelle turnover characterized by the formation of autophagosomes. ${ }^{25}$ Traditionally considered only as a survival mechanism for providing nutrients to cells under stress, prolonged autophagy is now considered to be an alternative form of cell death. Apoptosis is a tightly regulated process that is involved in tissue homeostasis, development and the removal of infected or damaged cells. ${ }^{26}$ This process is characterized by chromatin condensation, membrane blebbing, exposure of phosphatidyl serine on the outer leaflet of the plasma membrane and formation of apoptotic bodies. ${ }^{26}$ There is evidence that BNIP3 induces all three types of cell death (Figure 3).

When BNIP3 is overexpressed or induced following a stress stimuli in cells, it localizes to the mitochondria mediated by its TM domain. $^{2}$ This domain integrates into the outer membrane of the mitochondria, causing increase in ROS, opening of the PT pore and loss of $\Delta \psi \mathrm{m}$. When BNIP3 contains a mutation in its TM domain that prevents homodimerization but retains mitochondrial localization, it still induces cell death. ${ }^{4}$ In addition, replacing the TM domain with the Bcl-2 TM domain still localized BNIP3 to the mitochondria and had no effect on the cell death activity of BNIP3. ${ }^{4}$ This suggests that homodimerization is not required for BNIP3 function. The type of mitochondrial dysfunction induced by BNIP3 is generally agreed upon in the literature, but can be cell type specific. In cardiomyocytes, localization of BNIP3 to the mitochondria causes the release of cytochrome c leading to the activation of caspases and apoptosis. ${ }^{27}$ In neurons, BNIP3 is implicated in the release of endonuclease $\mathrm{G}$ from the mitochondria but not cytochrome c. ${ }^{28}$ This leads to a caspase-independent cell death. In epithelial-derived cells, overexpression of BNIP3 fails to release proteins from the mitochondria and fails to

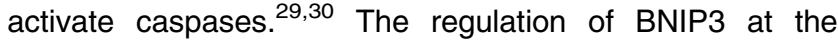
mitochondria is not well understood; however, some evidence gives insight to how BNIP3 might regulate mitochondrial function. In murine fibroblasts lacking Bax and Bak, BNIP3 failed to induce mitochondrial dysfunction and cell death. ${ }^{31}$ Upon restored expression of Bax and Bak, BNIP3 was able to induce ROS, loss of $\Delta \psi \mathrm{m}$ and release of mitochondrial cytochrome $\mathrm{c}$. This indicates that Bax and Bak cooperate with BNIP3 to induce cell death at the mitochondria. ${ }^{31}$ In isolated mitochondria, the C-terminal tail of BNIP3 is critical to the induction of mitochondrial loss of membrane potential and 
cytochrome $\mathrm{c}$ release. ${ }^{32}$ This indicates that proteinprotein interactions could alter BNIP3 mitochondrial function. Binding of $\mathrm{Bcl}-\mathrm{X}_{\mathrm{L}}$ to the TM domain of BNIP3 prevents BNIP3induced cell death. ${ }^{4}$ BNIP3 also binds to acetyl-coenzyme $A$ acyltransferase 2 (ACAA2) at the mitochondria and overexpression of ACAA2 blocks BNIP3-induced cell death in hepatocellular carcinoma cells and osteosarcoma cells. $^{33}$ Thus, regulation of BNIP3 at the mitochondria is critical for BNIP3-induced cell death, but the functional consequences in the mitochondria might differ depending on the cell type.

Electron microscopy (EM) images of epithelial-derived HEK293 cells expressing BNIP3 show evidence for necrosis-like cell death. Cells contain slightly dispersed foci of chromatin condensation, swollen mitochondria and extensive cytoplasmic vacuolation. ${ }^{29}$ Overexpression of BNIP3 can also induce autophagic cell death, measured by observation of autophagic vacuoles by EM, localization and processing of light chain 3 (LC3), which is a protein that is incorporated in autophagic membranes upon formation. ${ }^{16,12,18,34}$ BNIP3induced cell death is blocked by an inhibitor of autophagy (3-methyladenine), but not by an inhibitor of apoptosis (N-CBZ-Val-Aal-Asp(O-Me) fluoromethyl ketone). Knockdown of BNIP3 blocked hypoxia-induced autophagy and cell death, but did not block localization of LC3 with autophagosomes, therefore suggesting that BNIP3 is involved in autophagosome-lysosome fusion. ${ }^{34}$ Conversely, BNIP3 expression was induced early before the conversion of LC31 to LC3-II, and knockdown of BNIP3 could prevent LC3 conversion and autophagic cell death. ${ }^{35}$ BNIP3 is also induced by arsenic trioxide, ${ }^{12}$ ceramide $^{16}$ or concanavalin $A,{ }^{35}$ and concurrent induction of autophagy and cell death is mediated by BNIP3 in response to these stimuli. Loss of $\mathrm{Rb}$ has also been found to induce BNIP3-dependent autophagic cell death. ${ }^{18}$ How BNIP3 induces autophagy is not well understood, but recent evidence has led to the development of multiple theories (Figure 3). One model is based on the evidence that BNIP3 competes with Beclin-1 for binding of $\mathrm{Bcl}-2$. Bcl-2 blocks the induction of autophagy by binding to Beclin-1; therefore, when more BNIP3 is present to bind to $\mathrm{Bcl}-2$, beclin-1 is free to induce autophagy. ${ }^{36}$ In addition, BNIP3 has been found to cause damage to mitochondria in many different cell types. For example, BNIP3 damages mitochondria in cardiomyocytes as a result of ischemic injury leading to induction of autophagy. ${ }^{37}$ Another theory is that BNIP3 blocks autophagy by inhibiting mammalian target of rapamycin (mTOR) signaling through binding to Rheb (an activator of mTOR). ${ }^{38}$ The molecular mechanism of the involvement of BNIP3 in the induction of autophagy remains to be defined.

The most well-defined type of programmed cell death is apoptosis. Overexpression of BNIP3 in cardiomyocytes induces loss of $\Delta \psi \mathrm{m}$, ROS production, DNA condensation, activation of Bax and Bak and caspase activation, characteristic of an apoptotic response. ${ }^{27,31,39}$ However, other groups have failed to detect BNIP3-induced caspase activation and have observed some necrotic and autophagic cell death characteristics in cardiomyocytes. In photodynamic therapyresistant cell lines and effector cytotoxic T cells, expression of BNIP3 correlates with increased caspsase-independent cell death following cisplatin or activation-induced cell death. ${ }^{40,41}$ These results generally support the hypothesis that BNIP3 induces cell death in a caspase-independent manner and most likely only amplifies the apoptotic response in combination with other forms of cell death, such as necrosis and autophagy.

\section{Does BNIP3 expression play a role in heart disease?}

A coronary artery occlusion results in the deprivation of blood flow to the heart; this is termed ischemia. Ischemia is detrimental to cardiac myocytes because they require a constant supply of nutrients, which are limited under ischemic conditions. A pathological feature of prolonged ischemia is the induction of necrosis and apoptosis in cardiac myocytes and vascular cells of the heart. ${ }^{30,42}$ Ischemia is associated with hypoxia because there is a loss of blood flow to the tissues surrounding the blockage, and acidosis due to an increase in glycolysis and lactic acid production as a result of compensation for the lack of oxygen. Hypoxia-acidosis-induced cell death in cardiac myocytes has been found to be mediated by BNIP3. ${ }^{30}$ BNIP3 expression is upregulated during early ischemia and persists throughout reperfusion in rat ventricular myocytes. ${ }^{43}$ Moreover, high levels of BNIP3 protein have been detected in vivo in animal models of chronic heart failure. ${ }^{27,44}$ This gives strong evidence for a role of BNIP3 in heart disease.

Hypoxia has been observed to induce expression of BNIP3 and cell death in cardiac myocytes. Neonatal cardiac myocytes in culture show around $30 \%$ cell death when subjected to cycles of hypoxia-reoxygenation that mimic in vivo ischemia-reperfusion (I/R). ${ }^{45}$ Other reports observed neonatal myocyte cell loss after $24-72 \mathrm{~h}$ of hypoxia. ${ }^{46}$ In contrast, one study found that rapidly contracting neonatal cardiac myocytes have very minimal cell death for up to 5 days under hypoxic conditions. ${ }^{47}$ Nevertheless, neonatal cardiac myocytes have been identified to be more resistant to hypoxia than adult cardiac myocytes, demonstrated by the significant myocardial apoptosis in adult mice subjected to hypoxia. ${ }^{48,49}$ Overexpression of BNIP3 in a conditional mouse model was sufficient to induce myocardial apoptosis without subsequent stress. ${ }^{48}$ These mice were viable but had enlarged hearts that had progressive ventricular dilation, diminished systolic performance and contractile dysfunction at 10 weeks. This suggests that BNIP3 regulates hypoxia-induced cell death in cardiac myocytes.

In addition to hypoxia, acidosis may play a role in BNIP3induced cell death. ${ }^{30,50}$ Investigations into the function of BNIP3 in ischemia used cardiac myocytes cultured under hypoxic conditions, with high glucose content in the media to parallel the conditions induced by myocardial ischemia. ${ }^{51}$ Acidosis has been found to increase the stability of BNIP3 and its association with the mitochondria in cardiac myocytes. ${ }^{30,51}$ One group reports that cellular acidosis is essential for BNIP3 to induce cell death in cardiomyocytes. ${ }^{30,51}$ In contrast, other studies report that overexpression of BNIP3 induced apoptosis in cardiomyocytes independent of acidosis. ${ }^{45,52}$ The relative importance of acidosis and hypoxia in BNIP3-induced cell death remains to be determined. 
When blood flow returns to the areas of hypoxia (called reperfusion), which can occur spontaneously or through active clinical treatment, the hypoxic cells are subject to further damage induced by oxidative stress. ${ }^{50,53}$ Myocardial infarction, defined as an I/R event, if severe enough, leads to remodeling of the ventricle and subsequent heart failure. BNIP3 has been found to play a role in cardiac remodeling. ${ }^{54}$ There is evidence that BNIP3 could act as a sensor of oxidative stress in ventricular remodeling, where an increase in ROS induces homodimerization and activation of BNIP3 through a conserved cysteine residue. ${ }^{7}$ In mice where BNIP3 is knockout $(\mathrm{KO})$ in the heart, there was no observed effect on size, structure or contractile performance of the heart. ${ }^{48}$ BNIP3 ablation did not reduce infarct size after I/R, but did reduce post-I/R myocardial apoptosis by $50 \%$ in the peri-infarct region and minimized ventricular dilation, suggesting that BNIP3 is an important factor in ventricular remodeling. The role of reperfusion and cellular remodeling in BNIP3-induced cell death needs to be further evaluated.

The mode of cell death induced by BNIP3 in cardiac myocytes is debated in the literature, but most studies agree that inhibition of the mitochondrial PT pore opening inhibits BNIP3-induced cell death. Some studies report that BNIP3 induces a necrosis-like cell death where BNIP3-induced cell death fails to activate caspases $(3,8$ or 9$)$ and cell death is not blocked by caspase inhibitors ZVAD-fmk or Boc-D. ${ }^{30,50}$ Other studies report that caspase inhibitors suppress BNIP3induced cell death, suggesting that BNIP3 triggers an apoptotic-like cell death. ${ }^{27,37}$ Necrotic, autophagic and apoptotic features could also occur simultaneously, but further investigation is needed to determine the exact mechanism of BNIP3-induced cell death in the heart.

BNIP3 induces autophagy, but some model systems in I/R contend that this is a survival response. Hamacher-Brady et al. ${ }^{37}$ report that BNIP3 contributes to I/R injury in the ex vivo rat heart leading to induction of autophagy that could be blocked by dominant-negative BNIP3. Overexpression of ATG5 resulted in enhanced autophagy in BNIP3-overexpressing cells and protected them from BNIP3-induced cell death. Conversely, overexpressing a dominant-negative ATG5 that suppresses autophagosome formation leads to increased BNIP3-mediated cell death. ${ }^{37}$ Overexpression of Beclin 1 protected against BNIP3-induced cell death in HL-1 cells, also giving evidence for the protective role of autophagy in I/R-induced injury. ${ }^{39}$ These studies indicate that the initiation of autophagy by BNIP3 is protective initially following an ischemic event, but prolonged autophagy in the damaged heart preceding ischemic injury could contribute to autophagic cell death, or other forms of cell death may predominate over time.

The BNIP3 homolog NIX also plays a role in heart disease, but BNIP3 and NIX are reported to be regulated by two different pathways in the heart. ${ }^{17,55}$ BNIP3 expression is increased by the activation of transcription factors HIF-1 and $\mathrm{E} 2 \mathrm{~F}-1$, whereas NIX expression is regulated by activation of a $\mathrm{G}$ alpha(q) protein and protein kinase $\mathrm{C}$ alpha. ${ }^{52}$ This may explain why NIX cannot compensate for the loss of BNIP3 because it has an entirely different function and regulation in the heart. ${ }^{17,55}$

\section{Does BNIP3 play a role in cancer?}

BNIP3 expression has been identified to be elevated or suppressed in cancer. In addition, BNIP3 can be localized primarily in the nucleus or the cytoplasm, and its location varies between tumor types. There are multiple mechanisms that could control BNIP3 expression localization and function in tumors that are discussed below.

Elevated BNIP3 expression in tumors. Expression of BNIP3 is higher in many tumor types compared with matched normal tissue. The upregulation of BNIP3 expression is due to the presence of hypoxic regions in solid tumors. Hypoxia plays a major role in the malignant progression of tumors. Patients whose tumors contain hypoxic regions have a significantly worse prognosis when compared with patients with oxygenated tumors.

The expression of BNIP3 is upregulated in primary prostate tumors, ${ }^{56}$ glioblastoma multiforme (GBM) tumors, ${ }^{57}$ endometrial cancer, ${ }^{58}$ cervical tumors, ${ }^{59}$ ductal carcinoma in situ (DCIS), ${ }^{60}$ invasive breast carcinomas, ${ }^{61}$ lung tumors, ${ }^{62}$ ependymoma, ${ }^{63}$ follicular lymphomas ${ }^{64}$ and gastric adenocarcinomas. $^{65}$ (Table 1). Increased BNIP3 expression in these tumor types is also correlated with more aggressive tumors. BNIP3 upregulation in cervical tumors was correlated with more advanced clinical stages. ${ }^{59}$ In DCIS, BNIP3 expression levels increased with increasing grade, invasion level and the presence of invasive disease. ${ }^{60} \mathrm{~A}$ contrast is observed in murine breast cancer cell lines, where low BNIP3 expression in response to hypoxia leads to an increased metastatic potential to lung, liver and bone. ${ }^{66}$ In addition, BNIP3 upregulation in lung tumors, endrometrial cancer and ependymoma was associated with poor patient prognosis. ${ }^{58,62,63}$ BNIP3 is upregulated in lung cancer cell lines overexpressing p53 and is significantly higher in cell lines with high metastasis ability. ${ }^{67}$ BNIP3 expression was studied in a prostate cancer model, where a LNCaP progression cell line has lost PSA and androgen receptor (AR) expression and has increased metastasis and growth in xenografts of castrated mice. BNIP3 is upregulated in the cell lines that are further along in the progression of the disease, and are more

Table 1 Upregulation of BNIP3 in primary tumors

\begin{tabular}{|c|c|c|c|}
\hline Tumor type & $\begin{array}{l}\text { \% of primary tumor } \\
\text { samples with } \\
\text { upregulation }\end{array}$ & $\begin{array}{l}\text { Total } \\
\text { tumors }\end{array}$ & Reference \\
\hline $\begin{array}{l}\text { Gastric } \\
\text { adenocarcinomas }\end{array}$ & 67 & 48 & Lee et al. ${ }^{65}$ \\
\hline DCIS & 70 & 81 & Tan et al. ${ }^{61}$ \\
\hline Invasive breast & 78 & 251 & Tan et al. ${ }^{61}$ \\
\hline $\begin{array}{l}\text { carcinoma } \\
\text { Folicular } \\
\text { lymphomas }\end{array}$ & 77 & 35 & Sington et al. ${ }^{64}$ \\
\hline $\begin{array}{l}\text { Glioblastoma } \\
\text { multiforme }\end{array}$ & 97 & 48 & Burton et al. ${ }^{57}$ \\
\hline Lung tumors & 60 & 105 & $\begin{array}{l}\text { Giatromanolaki } \\
\text { et al. }{ }^{62}\end{array}$ \\
\hline Cervical tumors & 82 & 50 & Leo et al. ${ }^{59}$ \\
\hline Endometrial tumors & 32 & 72 & $\begin{array}{l}\text { Giatromanolaki } \\
\text { et al. }\end{array}$ \\
\hline
\end{tabular}


Table 2 Localization of BNIP3 in tumors

\begin{tabular}{|c|c|c|c|c|c|}
\hline Tumor type & $\begin{array}{l}\% \text { of tumors with } \\
\text { only nuclear BNIP3 }\end{array}$ & $\begin{array}{l}\% \text { of tumors with only } \\
\text { cytoplasmic BNIP3 }\end{array}$ & $\begin{array}{l}\% \text { of tumors with } \\
\text { both }\end{array}$ & $\begin{array}{l}\text { Total tumors } \\
\text { assayed }\end{array}$ & References \\
\hline Lung & 6 & 30 & 10 & 105 & Giatromanolaki et al. ${ }^{62}$ \\
\hline Gastric adenocarcinomas & 0 & 100 & 0 & 48 & Lee et al. ${ }^{65}$ \\
\hline Follicular lymphomas & 0 & 77 & 40 & 35 & Sington et al. ${ }^{64}$ \\
\hline Endometrial & 0 & 32 & 0 & 72 & Giatromanolaki et al. ${ }^{58}$ \\
\hline Pancreatic & 32 & 50 & 0 & 22 & Ishida et al. ${ }^{71}$ \\
\hline DCIS & 6 & 40 & 25 & 81 & Tan et al. ${ }^{61}$ \\
\hline Invasive breast carcinoma & 6 & 46 & 26 & 251 & Tan et al. ${ }^{61}$ \\
\hline GBM & 33 & 38 & 27 & 48 & Burton et al. ${ }^{57}$ \\
\hline Prostate & 11 & 27 & 56 & 149 & Shaida et al. ${ }^{56}$ \\
\hline
\end{tabular}

metastatic and androgen independent. ${ }^{68}$ BNIP3 expression in colorectal adenocarcinomas correlated with HIF1 levels, and both HIF1 and BNIP3 expression were also correlated with increased invasion and metastasis to the lymph nodes. ${ }^{69}$ Overall, elevated BNIP3 expression in tumors is associated with more aggressive disease.

These expression profiles of BNIP3 present a paradox in our understanding of BNIP3 function. Generally, overexpression of BNIP3 induces cell death, but in cancer, overexpression of BNIP3 is found in viable cells and is associated with poor prognosis. One potential explanation for this paradox is that the ability of BNIP3 to induce cell death has been blocked. Indeed, overexpression of $\mathrm{Bcl}-2$ found in many tumors also blocks BNIP3-induced cell death. Increased expression of epidermal growth factor (EGF) receptor is also found in many tumor types, and treating cancer cells with EGF blocks BNIP3-induced cell death (Figure 2). ${ }^{10}$ Furthermore, it is observed that BNIP3 does not localize to the mitochondria in tumors. The mechanism for blockade of BNIP3-induced cell death in tumors remains to be determined.

Silencing of BNIP3 expression in tumors. The bnip3 gene has been found to be silenced in tumors by epigenetic mechanisms, such as hypermethylation of the promoter and histone deacetylation. Lower BNIP3 expression than normal controls is observed in cell lines and primary tumor cells. Downregulation of BNIP3 results in a failure of tumor cells to undergo cell death and is associated with a chemoresistant phenotype and decreased patient survival. Restoration of BNIP3 expression, on the other hand, increases the sensitivity of tumor cells to drug treatment. Silencing BNIP3 expression could therefore be selected for by the cancer cells to evade cell death induced by chemotherapeutic drug treatment and harsh tumor microenvironments, such as hypoxia. This is in direct contrast with the tumors in which there is upregulation of BNIP3, where increased BNIP3 is a marker of more aggressive disease. Nevertheless, this gives supports to the concept that BNIP3-induced cell death is inhibited in tumors. The tumors that exhibit reduced BNIP3 expression and the current known mechanisms for silencing BNIP3 are discussed below.

Multiple pancreatic cell lines were discovered to silence the bnip3 gene by methylation of the promoter determined by bisulfite-restriction analysis. ${ }^{70}$ The bnip3 promoter is located within a 1700-bp CpG island spanning from -1162 to $+538 .^{70}$ When pancreatic cell lines that contain methylated bnip3 promoters were exposed to hypoxia, there was no induction of BNIP3 expression, and these cells are resistant to cell death. ${ }^{70}$ 5-Aza-2-deoxycytidine (Aza-dC) treatment reverses the methylation of the bnip3 gene, restores BNIP3 mRNA and protein expression and sensitizes the pancreatic cancer cells to hypoxia-mediated cell death. ${ }^{70} \mathrm{~A}$ report on primary pancreatic tumor tissue found that $18 \%$ of tumors were negative for BNIP3 staining, and that the clinical outcome negatively correlates with BNIP3 expression in tumors. ${ }^{71}$ Other studies on pancreatic ductal adenocarcinoma (PDAC) samples report that 80 and $90 \%$ of cases had decreased BNIP3 expression compared with normal pancreas. $^{70,71}$ Shorter patient survival correlated with low BNIP3 expression. ${ }^{72}$ Knocking down BNIP3 expression with siRNA resulted in resistance to fluorouracil (5-FU) and gemcitabine, the most common chemotherapeutic treatments for patients with PDAC. ${ }^{72}$ S100A4 has been reported to inhibit BNIP3 expression in PDAC cell lines that have unmethylated BNIP3 promoters, also contributing to gemcitabine resistance. ${ }^{21}$ Other studies found no association between BNIP3 expression and chemosensitivity of pancreatic cancer cell lines to gemcitabine. ${ }^{71}$

Some colon and gastric cancer cell lines do not induce BNIP3 under hypoxia due to methylation of the bnip3 promoter. ${ }^{73-75}$ Treatment with Aza-dC restores gene expression and the susceptibility to hypoxia-induced cell death. ${ }^{73}$ BNIP3 is downregulated in an oxaliplatin-resistant colon cancer cell line compared with the sensitive cell line identified by microarray, ${ }^{76}$ and in 5 -FU-resistant colorectal cells, ${ }^{77}$ suggesting that BNIP3 is an important regulator in the induction of drug-induced cell death in colon cancer. Some colon cancer cell lines have unmethylated bnip3 gene but still have no induction of BNIP3 expression under hypoxic conditions. In these cell lines, as well as in some methylated bnip3 gene cell lines, hypoxic BNIP3 expression was rescued by inhibiting HDAC activity. In primary colorectal cancer samples, 64\% had less BNIP3 expression in the tumor compared with normal control. ${ }^{74}$ Another study found that $66 \%$ of primary colorectal tumor samples and $49 \%(n=73)$ of primary gastric cancer tumor samples contained methylated promoter for bnip3, thereby downregulating BNIP3. ${ }^{75}$

Hepatocellular carcinoma (HCC) tumors also have bnip3 promoter hypermethylation. The more aggressive subclass $A$ HCC showed a higher level of bnip3 promoter methylation than the less aggressive $B$ type. ${ }^{72}$ bnip3 gene was found to be methylated in multiple myeloma (MM) patient samples and 
was significantly correlated with poor patient survival rates. Trichostatin A (HDAC inhibitor) and Aza-dC treatment of MM cell lines lead to an increase in the expression of BNIP3. ${ }^{78}$ Promoter methylation and downregulation of bnip3 expression is also observed in essential thrombocythemia patients and hematopoietic tumors compared with normal cells. ${ }^{79,80}$ Methylation was detected in $15 \%$ of primary leukemia samples, $17 \%$ of primary acute myloid leukemia samples and $21 \%$ of primary MM samples. ${ }^{80}$ Normal matched tissue samples from patients with pancreatic, colorectal and gastric tumors did not show any methylation of the bnip3 promoter. $^{70,75}$ This suggests that normal cells do not control the expression of BNIP3 by methylation of the promoter, and that bnip3 promoter methylation may be cancer specific. Overall, repression of BNIP3 expression occurs in a wide variety of tumors through several different mechanisms. The role that this silencing plays in cancer progression is unknown.

Cellular localization of BNIP3 in tumor cells. BNIP3 is observed to be localized to the cytoplasm, the nucleus or the perinuclear space. BNIP3 is expressed in the nucleus in noncancerous primary human astrocyte cultures, normal brain sections ${ }^{57}$ and chondrocytes of brachial cartilage, and also in the cytoplasm in the normal bronchial epithelium. ${ }^{62}$ Expression in tumors has been observed to be exclusively nuclear, exclusively cytoplasmic or both nuclear and cytoplasmic. The amount of nuclear versus cytoplasmic BNIP3 expression in different tumor types is shown in Table 2 and discussed below.

Nuclear BNIP3 is detected in GBM tumors, ${ }^{57}$ nonsmall cell lung tumors, ${ }^{62}$ cervical tumors, ${ }^{59}$ breast tumors ${ }^{61}$ and prostate tumors, ${ }^{56}$ and a perinuclear BNIP3 staining pattern is observed in pancreatic tumors ${ }^{72}$ and colorectal adenocarcinomas. ${ }^{69}$ In prostate tumors, there is a correlation between cytoplasmic BNIP3 expression and Gleason score, age AR and GLUT1 expression, but nuclear BNIP3 did not correlate with any clinicopathological factors. ${ }^{56}$ Nuclear BNIP3 in invasive breast carcinomas was associated with a smaller tumor size, lower tumor grade and estrogen receptor positivity, but there was no association with survival. However, in DCIS, nuclear BNIP3 correlated with a threefold increase in the risk of recurrence and a shorter disease-free survival rate. ${ }^{61}$ Mean survival times from Kaplan-Meier analysis for patients with PDAC indicated that nuclear BNIP3 is a poor survival marker in these tumors. ${ }^{71}$ Nuclear BNIP3 also significantly correlates with poor prognosis in nonsmall cell lung cancer. ${ }^{62}$ The mechanism of localization of BNIP3 to the nucleus is currently unknown, but this may be selected for in the tumors (1) to sequester BNIP3 away from the mitochondria and block cell death and/or (2) to induce a nuclear function of BNIP3 to promote cell survival.

\section{Conclusion}

Since the discovery that BNIP3 is one of the strongest upregulated proteins in response to hypoxia, contradictory results have been reported on the exact function for BNIP3. It induces a necrotic-like cell death, autophagic cell death, apoptosis, combination of these forms of cell death or cell survival. However, from the literature, consensus has emerged that BNIP3 localizes to the mitochondria causing increased ROS and loss of $\Delta \psi \mathrm{m}$ leading to cell death. However, many questions still remain to be answered, such as connection and timing between BNIP3-induced apoptosis, necrosis and/or autophagy.

In diseases, the role that BNIP3 plays remains unclear. In animal models of heart disease, KO of BNIP3 fails to alter the acute cellular damage in the heart but appears to increase remodeling after ischemia/reperfusion. This may indicate that BNIP3 fails to initiate cell death responses but amplifies them over time. In contrast, alteration of BNIP3 expression and localization in tumors suggests that BNIP3 function is altered. The mechanisms for this are not well understood, but what is clear is that BNIP3 may be a marker of tumor aggressiveness. Future studies that determine the mechanisms of how BNIP3 contributes to the pathogenesis of disease will give insight into BNIP3-regulated cell death and novel targets for therapeutic interventions.

Acknowledgements. We are grateful to Dr Lorrie Kirshenbaum for critical review of this manuscript. TRB holds a Terry Fox Foundation Scholarship and SBG holds a Manitoba Research Chair. SBG's research is supported by a grant from Canadian Institute for Health Research MOP 64330.

1. Yasuda M, Theodorakis $P$, Subramanian T, Chinnadurai G. Adenovirus E1B-19K/BCL-2 interacting protein BNIP3 contains a $\mathrm{BH} 3$ domain and a mitochondrial targeting sequence. J Biol Chem 1998; 273: 12415-12421.

2. Chen G, Cizeau J, Vande Velde C, Park JH, Bozek G, Bolton J et al. Nix and Nip3 form a subfamily of pro-apoptotic mitochondrial proteins. J Biol Chem 1999; 274: 7-10.

3. Ray R, Chen G, Vande Velde C, Cizeau J, Park JH, Reed JC et al. BNIP3 heterodimerizes with $\mathrm{Bcl}-2 / \mathrm{Bcl}-\mathrm{X}(\mathrm{L})$ and induces cell death independent of a Bcl-2 homology $3(\mathrm{BH} 3)$ domain at both mitochondrial and nonmitochondrial sites. J Biol Chem 2000; 275: 1439-1448.

4. Cizeau J, Ray R, Chen G, Gietz RD, Greenberg AH. The $C$. elegans orthologue ceBNIP3 interacts with CED-9 and CED-3 but kills through a BH3- and caspase-independent mechanism. Oncogene 2000; 19: 5453-5463.

5. Bocharov EV, Pustovalova YE, Pavlov KV, Volynsky PE, Goncharuk MV, Ermolyuk YS et al. Unique dimeric structure of BNip3 transmembrane domain suggests membrane permeabilization as a cell death trigger. J Biol Chem 2007; 282: 16256-16266.

6. Sulistijo ES, MacKenzie KR. Sequence dependence of BNIP3 transmembrane domain dimerization implicates side-chain hydrogen bonding and a tandem Gxx G motif in specific helix-helix interactions. J Mol Biol 2006; 364: 974-990.

7. Kubli DA, Quinsay MN, Huang C, Lee Y, Gustafsson AB. Bnip3 functions as a mitochondrial sensor of oxidative stress during myocardial ischemia and reperfusion. $A m \mathrm{~J}$ Physiol Heart Circ Physiol 2008; 295: H2025-H2031.

8. Yasuda M, Han JW, Dionne CA, Boyd JM, Chinnadurai G. BNIP3alpha: a human homolog of mitochondrial proapoptotic protein BNIP3. Cancer Res 1999; 59: 533-537.

9. Sowter HM, Ratcliffe PJ, Watson P, Greenberg AH, Harris AL. HIF-1-dependent regulation of hypoxic induction of the cell death factors BNIP3 and NIX in human tumors. Cancer Res 2001; 61: 6669-6673.

10. Kothari S, Cizeau J, McMillan-Ward E, Israels SJ, Bailes M, Ens K et al. BNIP3 plays a role in hypoxic cell death in human epithelial cells that is inhibited by growth factors EGF and IGF. Oncogene 2003; 22: 4734-4744.

11. Sandau US, Handa RJ. Glucocorticoids exacerbate hypoxia-induced expression of the proapoptotic gene Bnip3 in the developing cortex. Neuroscience 2007; 144: 482-494.

12. Kanzawa T, Zhang L, Xiao L, Germano IM, Kondo Y, Kondo S. Arsenic trioxide induces autophagic cell death in malignant glioma cells by upregulation of mitochondrial cell death protein BNIP3. Oncogene 2005; 24: 980-991.

13. Prabhakaran K, Li L, Zhang L, Borowitz JL, Isom GE. Upregulation of BNIP3 and translocation to mitochondria mediates cyanide-induced apoptosis in cortical cells. Neuroscience 2007; 150: 159-167.

14. Zhang S, Zhang Z, Sandhu G, Ma X, Yang X, Geiger JD et al. Evidence of oxidative stressinduced BNIP3 expression in amyloid beta neurotoxicity. Brain Res 2007; 1138: 221-230.

15. Yook YH, Kang KH, Maeng O, Kim TR, Lee JO, Kang KI et al. Nitric oxide induces BNIP3 expression that causes cell death in macrophages. Biochem Biophys Res Commun 2004; 321: 298-305

16. Daido S, Kanzawa T, Yamamoto A, Takeuchi H, Kondo Y, Kondo S. Pivotal role of the cell death factor BNIP3 in ceramide-induced autophagic cell death in malignant glioma cells. Cancer Res 2004; 64: 4286-4293. 
17. Yurkova N, Shaw J, Blackie K, Weidman D, Jayas R, Flynn B et al. The cell cycle factor E2F-1 activates Bnip3 and the intrinsic death pathway in ventricular myocytes. Circ Res 2008; 102: 472-479.

18. Tracy K, Dibling BC, Spike BT, Knabb JR, Schumacker P, Macleod KF. BNIP3 is an $\mathrm{RB} / \mathrm{E} 2 \mathrm{~F}$ target gene required for hypoxia-induced autophagy. Mol Cell Biol 2007; 27 6229-6242.

19. Baetz D, Regula KM, Ens K, Shaw J, Kothari S, Yurkova N et al. Nuclear factor-kappaBmediated cell survival involves transcriptional silencing of the mitochondrial death gene BNIP3 in ventricular myocytes. Circulation 2005; 112: 3777-3785.

20. Shaw J, Zhang T, Rzeszutek M, Yurkova N, Baetz D, Davie JR et al. Transcriptional silencing of the death gene BNIP3 by cooperative action of NF-kappaB and histone deacetylase 1 in ventricular myocytes. Circ Res 2006; 99: 1347-1354.

21. Mahon PC, Baril P, Bhakta V, Chelala C, Caulee K, Harada T et al. S100A4 contributes to the suppression of BNIP3 expression, chemoresistance, and inhibition of apoptosis in pancreatic cancer. Cancer Res 2007; 67: 6786-6795

22. Ikeda R, Tajitsu Y, Iwashita K, Che XF, Yoshida K, Ushiyama M et al. Thymidine phosphorylase inhibits the expression of proapoptotic protein BNIP3. Biochem Biophys Res Commun 2008; 370: 220-224.

23. Graham RM, Thompson JW, Wei J, Bishopric NH, Webster KA. Regulation of Bnip3 death pathways by calcium, phosphorylation, and hypoxia-reoxygenation. Antioxid Redox Signal 2007; 9: 1309-1315.

24. Manka D, Millhorn DE. A potential molecular link between aerobic glycolysis and cancer. Cell Cycle 2006; 5: 343-344.

25. Edinger AL, Thompson CB. Death by design: apoptosis, necrosis and autophagy. Curr Opin Cell Biol 2004; 16: 663-669.

26. McConkey DJ. Biochemical determinants of apoptosis and necrosis. Toxicol Lett 1998; 99 157-168.

27. Regula KM, Ens K, Kirshenbaum LA. Inducible expression of BNIP3 provokes mitochondrial defects and hypoxia-mediated cell death of ventricular myocytes. Circ Res 2002; 91: 226-231.

28. Zhang Z, Yang X, Zhang S, Ma X, Kong J. BNIP3 upregulation and EndoG translocation in delayed neuronal death in stroke and in hypoxia. Stroke 2007; 38: 1606-1613.

29. Vande Velde C, Cizeau J, Dubik D, Alimonti J, Brown T, Israels S et al. BNIP3 and genetic control of necrosis-like cell death through the mitochondrial permeability transition pore. Mol Cell Biol 2000; 20: 5454-5468.

30. Kubasiak LA, Hernandez OM, Bishopric NH, Webster KA. Hypoxia and acidosis activate cardiac myocyte death through the Bcl-2 family protein BNIP3. Proc Natl Acad Sci USA 2002; 99: 12825-12830.

31. Kubli DA, Ycaza JE, Gustafsson AB. Bnip3 mediates mitochondrial dysfunction and cell death through Bax and Bak. Biochem J 2007; 405: 407-415.

32. Kim JY, Cho JJ, Ha J, Park JH. The carboxy terminal C-tail of BNip3 is crucial in induction of mitochondrial permeability transition in isolated mitochondria. Arch Biochem Biophys 2002; 398: 147-152.

33. Cao W, Liu N, Tang S, Bao L, Shen L, Yuan $\mathrm{H}$ et al. Acetyl-coenzyme A acyltransferase 2 attenuates the apoptotic effects of BNIP3 in two human cell lines. Biochim Biophys Acta 2008; 1780: 873-880.

34. Azad MB, Chen Y, Henson ES, Cizeau J, McMillan-Ward E, Israels SJ et al. Hypoxia induces autophagic cell death in apoptosis-competent cells through a mechanism involving BNIP3. Autophagy 2008; 4: 195-204.

35. Chang CP, Yang MC, Liu HS, Lin YS, Lei HY. Concanavalin A induces autophagy in hepatoma cells and has a therapeutic effect in a murine in situ hepatoma model. Hepatology 2007; 45: 286-296.

36. Zhang H, Bosch-Marce M, Shimoda LA, Tan YS, Baek JH, Wesley JB et al. Mitochondrial autophagy is a HIF-1-dependent adaptive metabolic response to hypoxia. J Biol Chem 2008; 283: 10892-10903.

37. Hamacher-Brady A, Brady NR, Logue SE, Sayen MR, Jinno M, Kirshenbaum LA et al. Response to myocardial ischemia/reperfusion injury involves Bnip3 and autophagy. Cell Death Differ 2007; 14: 146-157.

38. Li Y, Wang Y, Kim E, Beemiller P, Wang CY, Swanson J et al. Bnip3 mediates the hypoxiainduced inhibition on mammalian target of rapamycin by interacting with Rheb. J Biol Chem 2007; 282: 35803-35813

39. Hamacher-Brady A, Brady NR, Gottlieb RA, Gustafsson AB. Autophagy as a protective response to Bnip3-mediated apoptotic signaling in the heart. Autophagy 2006; 2: 307-309.

40. Zacal N, Espiritu M, Singh G, Rainbow AJ. Increased BNip3 and decreased mutant p53 in cisplatin-sensitive PDT-resistant HT29 cells. Biochem Biophys Res Commun 2005; 331: 648-657.

41. Wan J, Martinvalet D, Ji X, Lois C, Kaech SM, Von Andrian UH et al. The Bcl-2 family proapoptotic molecule, BNIP3 regulates activation-induced cell death of effector cytotoxic T lymphocytes. Immunology 2003; 110: 10-17.

42. Searle J, Kerr JF, Bishop CJ. Necrosis and apoptosis: distinct modes of cell death with fundamentally different significance. Pathol Annu 1982; 17 (Part 2): 229-259.

43. Pitts KR, Derry JM, Kerkof K, Lawrence WA, Toombs CF. Differentially regulated functional gene clusters identified during ischemia and reperfusion in isolated cardiac myocytes using coverslip hypoxia. J Pharmacol Toxicol Methods 2008; 57: 42-51.

44. Bruick RK. Expression of the gene encoding the proapoptotic Nip3 protein is induced by hypoxia. Proc Natl Acad Sci USA 2000; 97: 9082-9087.
45. Webster KA, Discher DJ, Kaiser S, Hernandez O, Sato B, Bishopric NH. Hypoxia-activated apoptosis of cardiac myocytes requires reoxygenation or a pH shift and is independent of p53. J Clin Invest 1999; 104: 239-252.

46. Long X, Boluyt MO, Hipolito ML, Lundberg MS, Zheng JS, O'Neill L et al. p53 and the hypoxia-induced apoptosis of cultured neonatal rat cardiac myocytes. J Clin Invest 1997 99: 2635-2643.

47. Webster KA, Bishopric NH. Molecular regulation of cardiac myocyte adaptations to chronic hypoxia. J Mol Cell Cardiol 1992; 24: 741-751.

48. Diwan A, Krenz M, Syed FM, Wansapura J, Ren X, Koesters AG et al. Inhibition of ischemic cardiomyocyte apoptosis through targeted ablation of Bnip3 restrains postinfarction remodeling in mice. J Clin Invest 2007; 117: 2825-2833.

49. Lee SD, Kuo WW, Wu CH, Lin YM, Lin JA, Lu MC et al. Effects of short- and long-term hypobaric hypoxia on Bcl2 family in rat heart. Int J Cardiol 2006; 108: 376-384.

50. Webster KA, Graham RM, Bishopric NH. BNip3 and signal-specific programmed death in the heart. J Mol Cell Cardiol 2005; 38: 35-45.

51. Graham RM, Frazier DP, Thompson JW, Haliko S, Li H, Wasserlauf BJ et al. A unique pathway of cardiac myocyte death caused by hypoxia-acidosis. J Exp Biol 2004; 207 (Part 18): $3189-3200$

52. Yussman MG, Toyokawa T, Odley A, Lynch RA, Wu G, Colbert MC et al. Mitochondrial death protein Nix is induced in cardiac hypertrophy and triggers apoptotic cardiomyopathy. Nat Med 2002; 8: 725-730.

53. Jennings RB, Steenbergen Jr C, Reimer KA. Myocardial ischemia and reperfusion. Monog Pathol 1995; 37: 47-80.

54. Whelan RS, Mani K, Kitsis RN. Nipping at cardiac remodeling. J Clin Invest 2007; 117 2751-2753.

55. Galvez AS, Brunskill EW, Marreez Y, Benner BJ, Regula KM, Kirschenbaum LA et al. Distinct pathways regulate proapoptotic Nix and BNip3 in cardiac stress. J Biol Chem 2006; 281: 1442-1448.

56. Shaida N, Launchbury R, Boddy JL, Jones C, Campo L, Turley $\mathrm{H}$ et al. Expression of BNIP3 correlates with hypoxia-inducible factor (HIF)-1alpha, HIF-2alpha and the androgen receptor in prostate cancer and is regulated directly by hypoxia but not androgens in cell lines. Prostate 2008; 68: 336-343.

57. Burton TR, Henson ES, Baijal P, Eisenstat DD, Gibson SB. The pro-cell death Bcl-2 family member, BNIP3, is localized to the nucleus of human glial cells: implications for glioblastoma multiforme tumor cell survival under hypoxia. Int J Cancer 2006; 118: 1660-1669.

58. Giatromanolaki A, Koukourakis MI, Gatter KC, Harris AL, Sivridis E. BNIP3 expression in endometrial cancer relates to active hypoxia inducible factor 1alpha pathway and prognosis. J Clin Pathol 2008; 61: 217-220.

59. Leo C, Horn LC, Hockel M. Hypoxia and expression of the proapoptotic regulator BNIP3 in cervical cancer. Int J Gynecol Cancer 2006; 16: 1314-1320.

60. Sowter HM, Ferguson M, Pym C, Watson P, Fox SB, Han C et al. Expression of the cell death genes BNip3 and NIX in ductal carcinoma in situ of the breast; correlation of BNip3 levels with necrosis and grade. J Pathol 2003; 201: 573-580.

61. Tan EY, Campo L, Han C, Turley H, Pezzella F, Gatter KC et al. BNIP3 as a progression marker in primary human breast cancer; opposing functions in in situ versus invasive cancer. Clin Cancer Res 2007; 13 (2 Part 1): 467-474.

62. Giatromanolaki A, Koukourakis MI, Sowter HM, Sivridis E, Gibson S, Gatter KC et al. BNIP3 expression is linked with hypoxia-regulated protein expression and with poor prognosis in non-small cell lung cancer. Clin Cancer Res 2004; 10: 5566-5571.

63. Lukashova-v Zangen I, Kneitz S, Monoranu CM, Rutkowski S, Hinkes B, Vince GH et al. Ependymoma gene expression profiles associated with histological subtype, proliferation, and patient survival. Acta Neuropathol 2007; 113: 325-337.

64. Sington J, Giatromanolaki A, Campo L, Turley H, Pezzella F, Gatter KC. BNIP3 expression in follicular lymphoma. Histopathology 2007; 50: 555-560.

65. Lee SH, Jeong EG, Yoo NJ. Mutational and expressional analysis of BNIP3, a proapoptotic Bcl-2 member, in gastric carcinomas. APMIS 2007; 115: 1274-1280.

66. Manka D, Spicer Z, Millhorn DE. Bcl-2/adenovirus E1B $19 \mathrm{kDa}$ interacting protein-3 knockdown enables growth of breast cancer metastases in the lung, liver, and bone. Cancer Res 2005; 65: 11689-11693.

67. Yan J, Yun $\mathrm{H}$, Yang $\mathrm{Y}$, Jing B, Feng $\mathrm{C}$, Song-bin F. Upregulation of BNIP3 promotes apoptosis of lung cancer cells that were induced by p53. Biochem Biophys Res Commun 2006; 346: 501-507.

68. Chen Q, Watson JT, Marengo SR, Decker KS, Coleman I, Nelson PS et al. Gene expression in the LNCaP human prostate cancer progression model: progression associated expression in vitro corresponds to expression changes associated with prostate cancer progression in vivo. Cancer Lett 2006; 244: 274-288.

69. Koukourakis MI, Giatromanolaki A, Polychronidis A, Simopoulos C, Gatter KC, Harris AL et al. Endogenous markers of hypoxia/anaerobic metabolism and anemia in primary colorectal cancer. Cancer Sci 2006; 97: 582-588.

70. Okami J, Simeone DM, Logsdon CD. Silencing of the hypoxia-inducible cell death protein BNIP3 in pancreatic cancer. Cancer Res 2004; 64: 5338-5346.

71. Ishida M, Sunamura M, Furukawa T, Akada M, Fujimura H, Shibuya E et al. Elucidation of the relationship of BNIP3 expression to gemcitabine chemosensitivity and prognosis. World $J$ Gastroenterol 2007; 13: 4593-4597.

72. Erkan M, Kleeff J, Esposito I, Giese T, Ketterer K, Buchler MW et al. Loss of BNIP3 expression is a late event in pancreatic cancer contributing to chemoresistance and worsened prognosis. Oncogene 2005; 24: 4421-4432. 
73. Ishiguro M, lida S, Uetake H, Morita S, Makino H, Kato K et al. Effect of combined therapy with low-dose 5-aza-2'-deoxycytidine and irinotecan on colon cancer cell line HCT-15. Ann Surg Oncol 2007; 14: 1752-1762.

74. Bacon AL, Fox S, Turley H, Harris AL. Selective silencing of the hypoxia-inducible factor 1 target gene BNIP3 by histone deacetylation and methylation in colorectal cancer. Oncogene 2007; 26: 132-141.

75. Murai M, Toyota M, Suzuki H, Satoh A, Sasaki Y, Akino K et al. Aberrant methylation and silencing of the BNIP3 gene in colorectal and gastric cancer. Clin Cancer Res 2005; 11 1021-1027.

76. Tang H, Liu YJ, Liu M, Li X. Establishment and gene analysis of an oxaliplatinresistant colon cancer cell line THC8307/L-OHP. Anticancer Drugs 2007; 18 633-639.
77. de Angelis PM, Fjell B, Kravik KL, Haug T, Tunheim SH, Reichelt W et al. Molecular characterizations of derivatives of $\mathrm{HCT} 116$ colorectal cancer cells that are resistant to the chemotherapeutic agent 5-fluorouracil. Int J Oncol 2004; 24: 1279-1288.

78. Heller G, Schmidt WM, Ziegler B, Holzer S, Mullauer L, Bilban M et al. Genome-wide transcriptional response to 5 -aza- $2^{\prime}$-deoxycytidine and trichostatin a in multiple myeloma cells. Cancer Res 2008; 68: 44-54.

79. Tenedini E, Fagioli ME, Vianelli N, Tazzari PL, Ricci F, Tagliafico E et al. Gene expression profiling of normal and malignant CD34-derived megakaryocytic cells. Blood 2004; 104: 3126-3135.

80. Murai M, Toyota M, Satoh A, Suzuki H, Akino K, Mita H et al. Aberrant DNA methylation associated with silencing BNIP3 gene expression in haematopoietic tumours. $\mathrm{Br} \mathrm{J}$ Cancer 2005; 92: 1165-1172. 